\title{
A strong adaptable autofocusing approach of off-axis infrared digital holography under different quality conditions of holograms
}

\author{
Ning Liu*, Chao Yang \\ Nanjing University of Posts and Telecommunications, School of Optoelectronics \\ Engineering, Nanjing, Jiangsu Province, 210023. \\ *Corresponding author: coolboy006@sohu.com
}

\begin{abstract}
In this paper, we present an innovative autofocusing criterion for the reconstruction of infrared digital holograms. This criterion has the advantages of fast, efficient and precision when determining the reconstruction distance of off-axis digital holography. This criterion is a mean-free high frequency calculation process. We focus on the problem of mean value drifting found in previous published methods and design our new approach to solve it. Unlike the previous methods perform well only with high quality holograms, our method is effective for both high and low quality holograms. Even when hologram is degraded by destructive interference, our method still performs well. This method helps to automatically determine the precise reconstruction distance, and we are sure that this technology can be applied in industrial applications in the future.
\end{abstract}

Keywords: autofocusing; infrared digital holography; various quality of holograms; strong adaptability

\section{Introduction}

In the digital holography process, many researchers have focused on how to search and determine the correct and accurate reconstruction distance of the image plane. Though it can be established during the optical setup by precise measurement tools or by repeated tests during the process of computer reconstruction, these approaches are cumbersome and very time-consuming. Autofocusing plays an important role in determining the correct reconstruction distance in digital holography[1], and many algorithms have flourished during these years[2-7]. These algorithms used the total 
sum of the gradient[4,6], the Laplacian[4,5], as well as the variance[4,7] of gray-value image distribution. Other methods mainly focused or were based on amplitude analysis[8,9], intensity gradient[10], self-entropy[11], local intensity variance[12], spectrum norms[13] and so on. Each of them achieved certain success in automatically searching the reconstruction distance. In the last three years, the autofocusing of digital holography has trended toward less computation, faster calculation speed and great adaptability. For example, P. Memmolo et al. proposed a new autofocusing approach by using the criterion of the "tamura coefficient" in 2011[14]. The procedure for this method is quite simple. It uses the square value of the standard deviation(STD) and the mean value of a reconstruction hologram. According to their discussion, this criterion works well in determining the focusing distance in the reconstruction process, and it is better than the Laplacian, variance and Gradient models. In 2014, they furthered their research by proposing a new autofocusing approach with the criterion of the "Gini” index[15]. This new approach is based on the gray-level norm calculation to determine the best reconstruction distance. They claimed that this new approach was better than the previous one but had the disadvantage of complex computations. A Turkish research group proposed a fast autofocusing method using scaled holograms in 2013[16]. They introduced a VAR criterion based on normalizing the variance of the image with its mean. Images that contain abnormally high gray-value values were assigned lower sharpness values. Meanwhile, a Chinese research group proposed a dual-illumination criterion in 2012[17] to achieve the same goal. Their method adapted two illumination beams as the object waves, and the criterion was set by calculating the root mean square (RMSE) of both the phases and amplitudes of the reconstruction images of these two beams. During our research, we found that these methods $[14,16,17]$ are applicable when automatically calculating the reconstruction distance because of their simplicity, fast calculation and precision. All the works gave great inspiration to our research. However, we have discovered two issues about the previous research: 1 . the adaptability under different quality conditions of holograms and 2. the extend of the experiments under the lab environment contribute to the future industrialization of the 
real world. According to the first issue, these methods[14,16,17] all use the mean value of the reconstruction image during the reconstruction procedure. That is to say, if the mean value does not drift during reconstruction, all the methods work well. But if the mean value drifts during reconstruction, unexpected results show up. Normally in the high-tech lab, the experiments are conducted with high quality equipment, and the problem of mean value drifting is very slight, but it does exist. The detailed analysis about mean value drifting will be discussed in the following sections. This has a certain connection with the second issue. Once digital holography technology is populated in every aspect of human lives, the capture of a hologram will be conducted in the open environment of the real world. Thus, the quality of the captured holograms cannot be compared to the ones captured in the high-tech labs. Hence, the adaptability of the autofocusing approach must be greatly improved to fit those holograms with different qualities. Besides, when it comes to the real world application, the gradient[4,6] and Laplacian[4,5] methods are too complex to calculate. Too much computation will lower the processing speed for the real-time application of industrialization.

In this paper, we present an autofocusing method with strong adaptability. First, we use an 850nm near infrared laser illumination and the off-axis optical setup of digital holography to capture the high quality holograms, and then we adjust the equipment and laser source to degrade the image quality. We combine the thought of the proposed mean-based and high frequency-based methods, and set up our new criterion to calculate the reconstruction distance. We test our method as well as the proposed methods on both high quality holograms and low quality holograms, and find that our method is more suitable for both kinds of images. During the experiment, we find that, even when destructive interference accidently joins in the reconstruction procedure, our method still works pretty well when determining the precise reconstruction distance. This method is innovative under different quality conditions of holograms, and could be applied in future industrialization.

\section{Description of the optical setup}

The optical setup of the experiment is quite simple. We use a traditional off-axis 
digital holography setup to illuminate a transparent mini statue and a MEMS chip to acquire both low quality and high quality holograms. The optical setup is shown as fig.1.

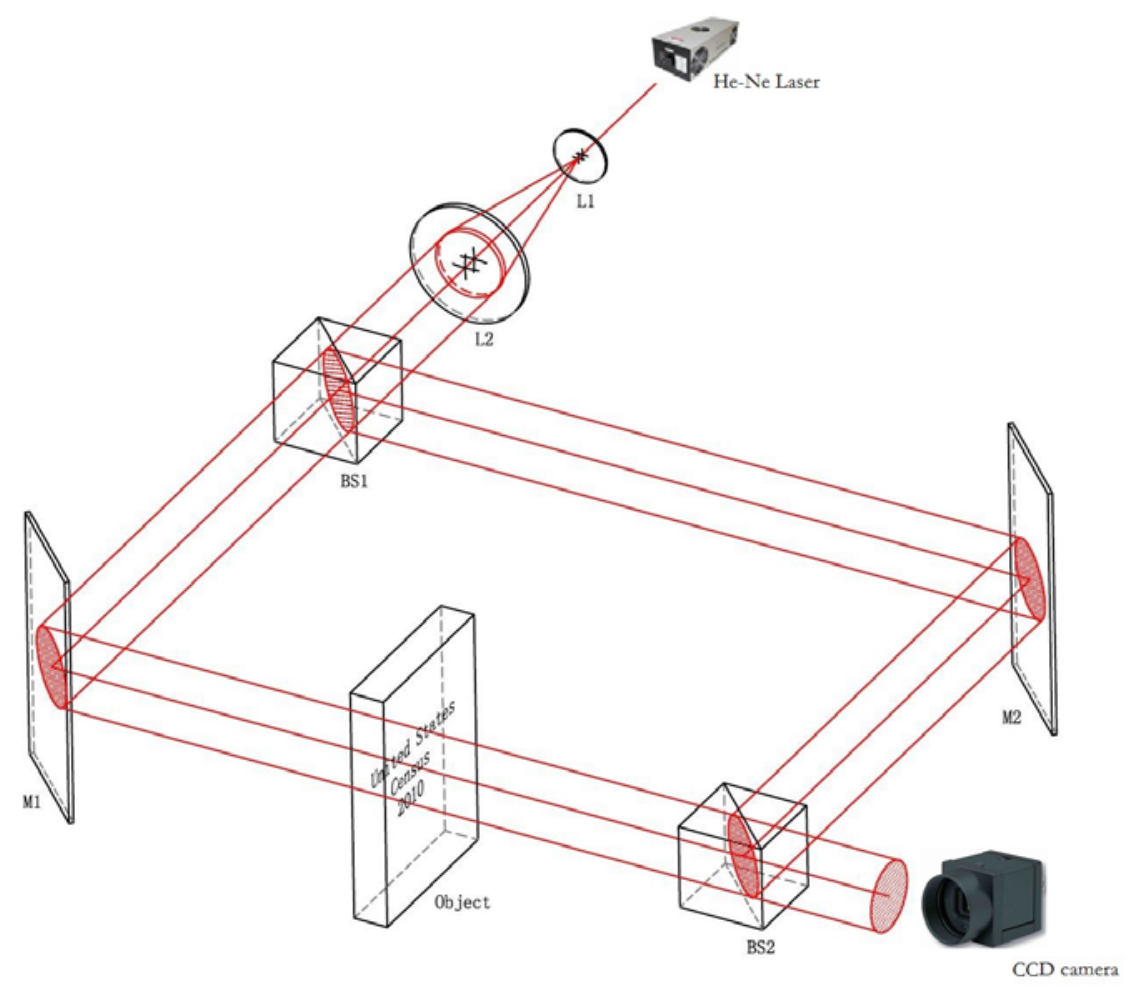

Fig.1 optical setup of the experiment

As in fig.1, the laser source we use is an $850 \mathrm{~nm}$ semiconductor laser with the power of 32mw. Focal lenses $\mathrm{L} 1(\mathrm{f} 1=6.2 \mathrm{~mm})$ and $\mathrm{L} 2(\mathrm{f} 2=150 \mathrm{~mm})$ form a beam expander. The two cubes are beam splitters. M1 and M2 are reflecting mirrors. The CCD has a pixel dimension of $1280 \times 1024$, and pixel pitch is $5.2 \mu \mathrm{m}$. When illuminating the MEMS chip, it locates in the position where M1 belongs. When illuminating the mini statue of "2010 United States Census", the expanded laser is shooting the letter "e" and " $\mathrm{n}$ " in the word "Census". In this experiment, we mainly focus on the amplitude holography. Although the mini statue is mostly transparent, the criterion is calculated based on the intensity of the reconstruction image. The phase demonstration is shown in the following figures, but it is not our main concern.

\section{Mean value drifting of reconstruction image}

As we mentioned above, recently published autofocusing methods[14,16,17] are usually based on the mean value calculation. These kinds of methods are more 
effective than the usual high frequency based methods such as the gradient $[4,6]$ and the Laplacian[4,6]. The mean value can be recognized as the average energy of an image. During the autofocusing process, all the estimated distances are chosen as the index $Z_{0}$ in the angular spectrum transfer function which is used to reconstruct the image. The reconstruction process can be described as the following functions[16]:

$$
\begin{aligned}
R(\mathrm{x}, \mathrm{y}) & =F^{-1}[F(\mathrm{I}(\mathrm{x}, \mathrm{y})) H(\mu, v)] \\
H(\mu, v) & = \begin{cases}e^{j k z_{0} \sqrt{1-(\lambda \mu)^{2}-(\lambda v)^{2}}}, & \sqrt{\mu^{2}+v^{2}}<1 / \lambda \\
0 & \text { otherwise }\end{cases}
\end{aligned}
$$

where $\mathrm{I}(\mathrm{x}, \mathrm{y})$ is the hologram image, $H(\mu, v)$ is the angular spectrum transfer function calculated for depth $Z_{0}, F$ is the Fourier transform and $R(\mathrm{x}, \mathrm{y})$ is the final reconstruction image. In recent published methods, no matter the "tamura coefficient", "VAR index" or the dual-illumination criterion, the mean value plays an important role in each one of them. Usually in the autofocusing criterion calculation, the STD and RMSE index use every gray value of the imaging pixel to subtract the mean value to determine the error between the current pixel value and the mean value. The total error will be summarized according to the dimension of the captured hologram, and then averaged by the dimension[14,17] or the mean value[16]. Then the criterion becomes available. During our research, we find that these kinds of criteria work pretty well when dealing with high quality holograms. This is because these holograms are "pure” and “clean”. Very little interference occurred, e.g., ones that usually come from the illumination source or the optical components are merged into the holograms. But unfortunately, when we seriously degrade the quality of the holograms, these proposed methods become more or less ineffective. When imaging with the low quality equipment, the hologram captures not only the object information but also the interference information. The interference is usually manifested as circle shaped clutters or stripes. All the interference will be accounted for in the criteria determination, and mislead the mean value calculation. Thus, the mean value becomes fluctuating and unstable. If this happens, the STD or RMSE index will be seriously affected because the error summary becomes uncontrollable. The following are some 
comparisons of the high quality holograms and low quality holograms, shown in fig.2.

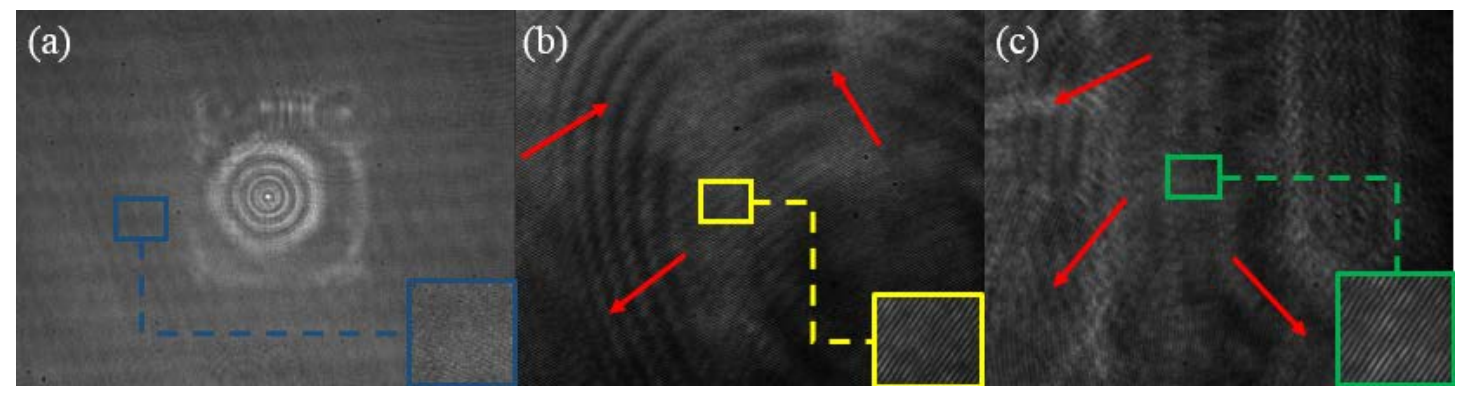

Fig.2 comparison of different quality holograms. (a)high quality hologram; (b),(c)low quality holograms with serious interference are marked by red arrows

The following table.1 is a root mean square(RMS) illustration of the reconstruted image of fig.1(a), (b) and (c) respectively at precise reconstruction distances. We choose a small flat region of the reconstruction images $(100 \times 100$ pixels $)$ to calculate the RMS value of them. The RMS value gives a clear definition of the quality of the chosen holograms.

Table.1 RMS definition of the hologram qualities

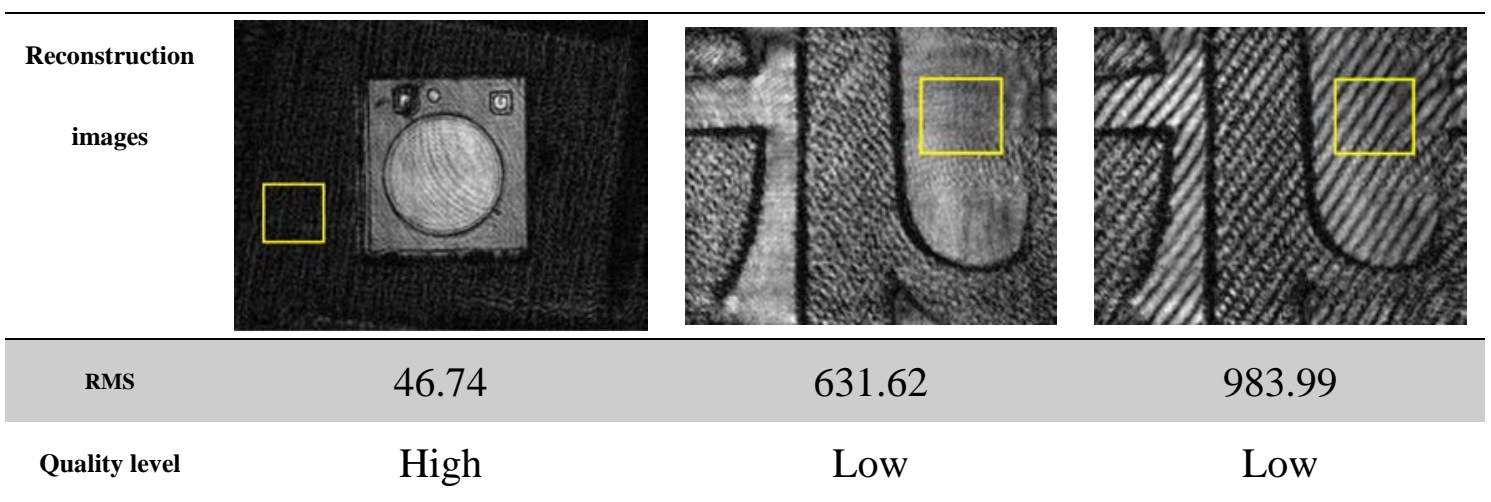

Next, we provide some data flow charts for the proposed criteria of "tamura coefficient" and "VAR index". These show the performance when dealing with both kinds of holograms. 

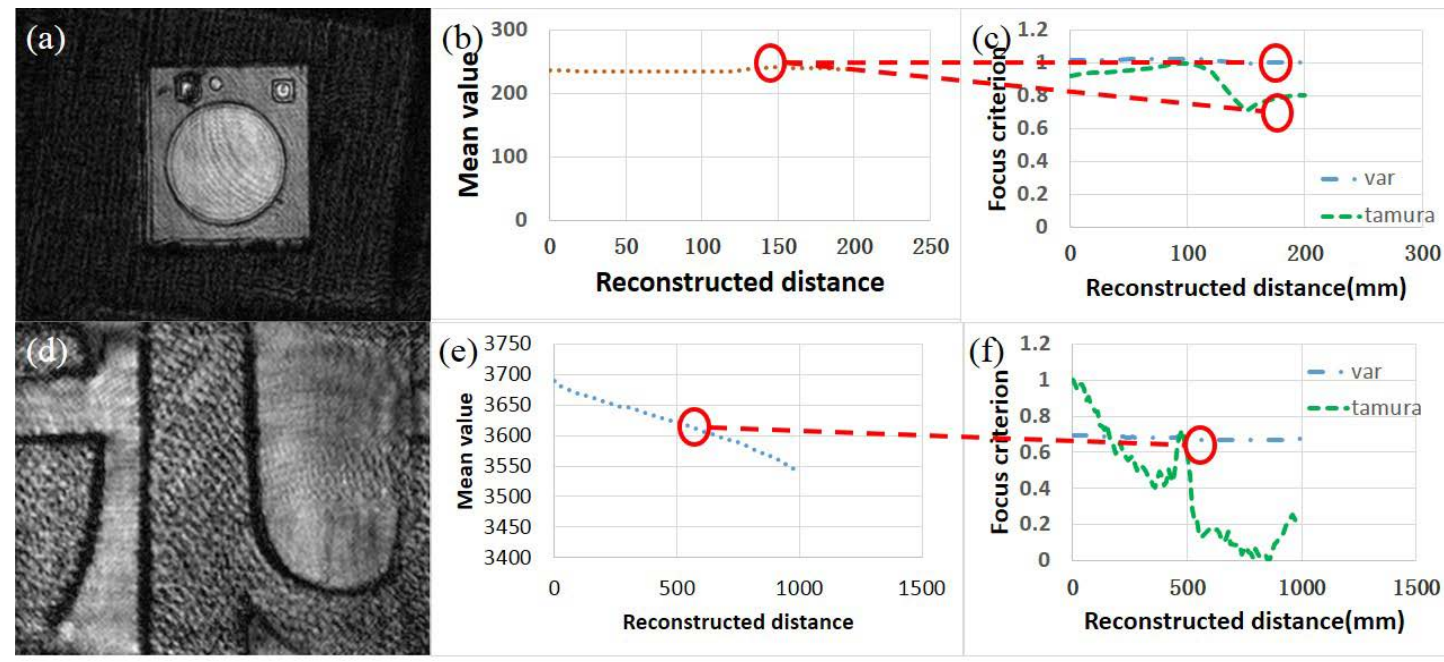

Fig.3 reconstruction images of high and low quality holograms with comparison flow charts. (a),(d)reconstruction images according to fig.1(a) and (c), respectively; (b),(e)mean value fluctuation on these two kinds of holograms; (c),(f)reconstruction criterion calculation using the proposed methods.

In fig.2 and fig.3, we can see that, for the high quality hologram, the mean value hardly fluctuates. The proposed criteria can easily and presicely determine the reconstruction distance of $140 \mathrm{~mm}$ which has been marked with red circles in fig.3(b) and (c). But results are unexpected when dealing with the low quality hologram. Due to the serious interference shown in fig.2(b) and fig.2(c) with red arrows, the mean value curve keeps on dropping during the reconstruction process in fig.3(b) and (e). Although the proposed criteria have peaks at the correct distance, this is still not enough for automatic determination by the computer. The peaks are neither the biggest nor the smallest value in the criteria curves. When this happens, the proposed methods become ineffective and lead to the failure of autofocusing.

\section{Our criterion with strong adaptability}

A good criterion should be adaptable enough to fit in any image quality situation. According to the analysis of mean value drifting, it is necessary to figure out a way to minimize the role of the mean value during the criterion calculation. Inspired by the gradient methods[4,6], we know that during the reconstruction process, the high frequency details of the image become more and more clear. These details contain the edge and shape information about the observing object. That means the high 
frequency energy of the image will become stronger when the precise reconstruction distance is reached. In fact, the high frequency energy is quite small compared to the mean value energy. So in the proposed methods, although the high frequency energy may lead to the correct judgment, it is still overwhelmed by the mean value energy. During our research, we have found a way to suppress the influence of the mean value, leaving only the high frequency energy in the calculation as follows:

$$
\begin{gathered}
I_{h i g h}(\mathrm{x}, \mathrm{y})=I(\mathrm{x}, \mathrm{y})-\hat{I} \\
\hat{I}_{\text {high }}=\frac{\sum_{x, y}\left|I_{h i g h}(\mathrm{x}, \mathrm{y})\right|}{M \times N} \\
R M S_{\text {high }}=\sqrt{\frac{\sum_{x, y}\left(\left|I_{h i g h}(\mathrm{x}, \mathrm{y})\right|-I_{\text {high }}\right)^{2}}{M \times N}} \\
\operatorname{cri}=\frac{R M S}{I_{\text {high }}^{\wedge}}
\end{gathered}
$$

where, $\hat{I}$ represents the mean value of the original hologram. $I_{\text {high }}(\mathrm{x}, \mathrm{y})$ represents the high frequency part of the hologram. $\mathrm{M}, \mathrm{N}$ represent the dimension of the captured image. As we see from eq.3-eq.6, our aim is to eliminate the mean value energy from the original hologram, leaving only the high frequency part to calculate. When the mean value has been subtracted, the RMS index of the high frequency component reflects the statistical distribution of the high frequency energy. When the correct reconstruction distance is reached, the distribution of high frequency energy will concentrated more and more towards the index $\hat{I}_{\text {high }}$. Besides, $\hat{I}_{\text {high }}$ will reach its upper peak when the reconstruction distance is reached, which causes the RMS index to reach its lower peak. The index cri from eq. 6 will then be calculated as the criterion which determines the precise reconstruction distance. We use eq.3-eq.5 onto the original hologram and eliminate the fluctuation caused by the mean value, and use eq. 1-eq.2 to reconstruct the hologram under every step-in distances. Eq.6 is then used to determine the energy focus of every reconstruction image, which make sure the correct reconstruction distance can be calculated during the autofocusing process. 
We have run lots of tests to prove that our criterion works not only as well as the proposed methods[14,16,17] for high quality holograms, but also much better and more effective for low quality holograms. First, we run our criterion with high quality holograms along with the "tamura coefficient" and "VAR index" using fig.2(a) to get the following results.
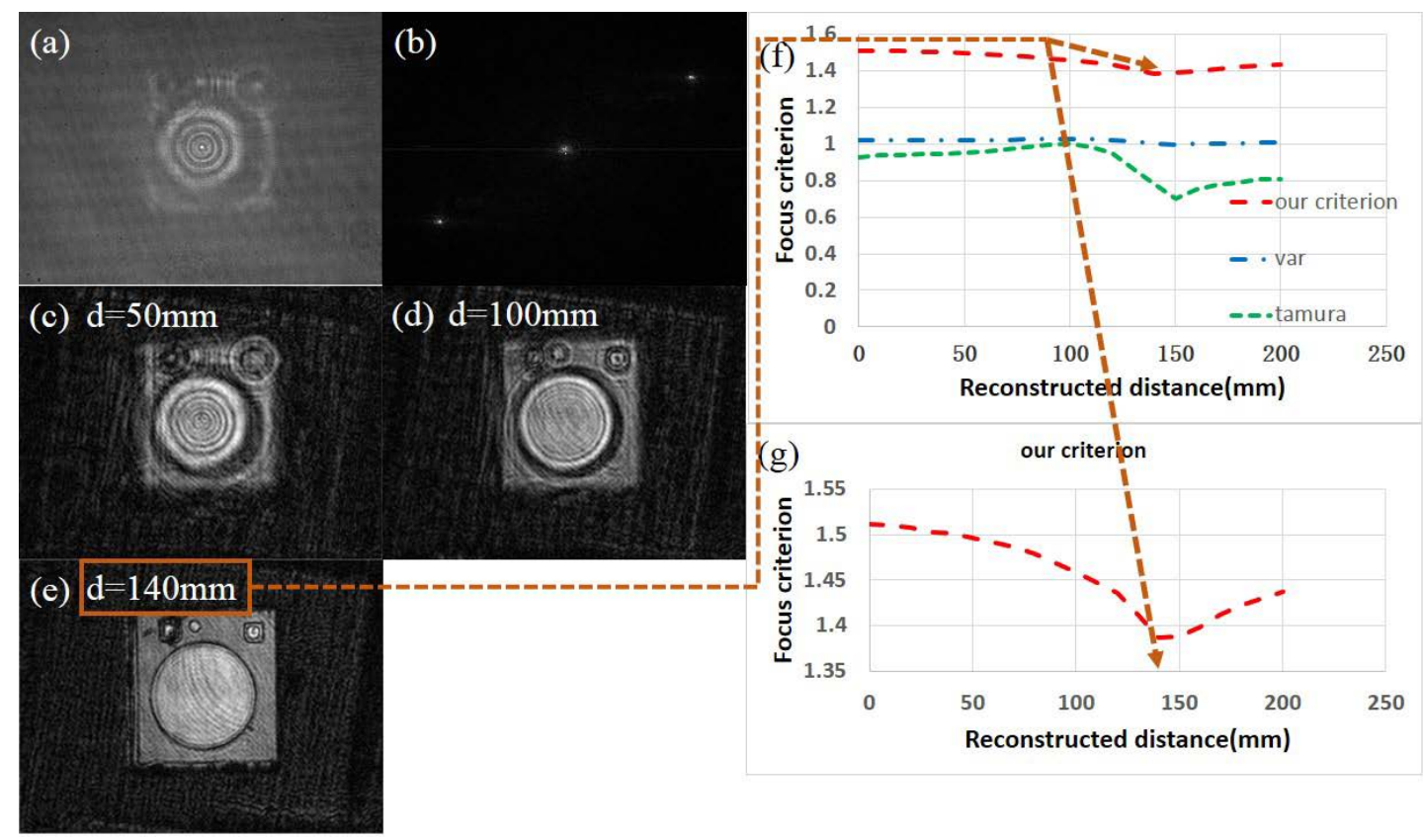

Fig.4 comparison between our criterion and the proposed methods using high quaility hologram. (a)original hologram; (b)Fourier transform of the original hologram; (c),(d),(e) reconstruction processes under different distances; (f)three autofocusing criterion curves with different methods; (g)curve detail of our criterion

We can see from fig.4 that with a high quality hologram, the three criteria can easily determine the correct reconstruction distance. Continuing the analysis of the comparison, the performance of the "VAR index" is a little bit inadequate, but still gives the correct result. Our criterion and the "tamura coefficient” clearly demonstrate the lowest peak for determination. This is because that, according to the analysis above, when there is no destructive interference, the calculation of high frequency energy and mean value energy produces almost the same results. Next, we apply these three criteria to the low quality hologram, and different results show up. 


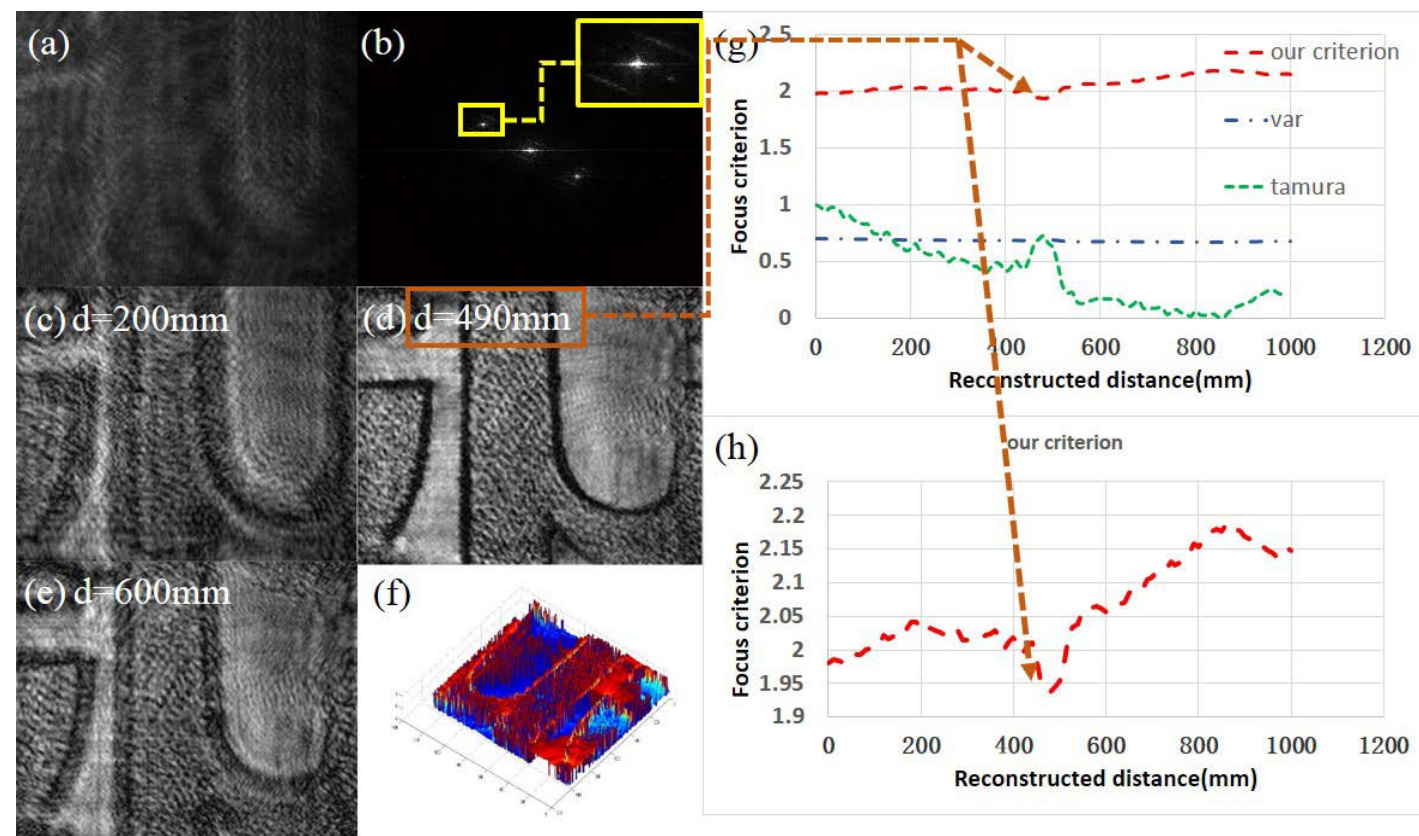

Fig.5 comparison between our criterion and the proposed methods using low quaility hologram. (a)original hologram; (b)Fourier transform of the original hologram; (c),(d),(e) reconstruction processes under different distances; (f)Pseudo-three-dimension plot of the phase of the reconstruction image of (d); (g)three autofocusing criterion curves with different methods; (h)curve detail of our criterion

In fig.5, the comparison results are apparently proved that our criterion is better to determine the correct reconstruction distance for a low quality hologram. In fig.5(g), the "VAR index" curve is almost a straight line because of the destructive interference. No distance can be calculated using this criterion. Meanwhile, although there is a peak in the curve of "tamura coefficient" at the correct distance, the computer cannot distinguish it from the whole curve because it is neither the uppermost peak nor the lowermost peak. But with our criterion, the peak shows up directly at the precise reconstruction distance and can be determined by the computer as the meanningful result.

During the test processes in our research, we found that, the adaptability of our criterion is far beyond what shows up in fig.5. We introduced a second illumination with the same wavelength but with different polarization state from the same laser source as another object wave to illuminate the object. That is to say, there are two object waves with different polarization directions illuminating the same object. In 
this experiment, the polarization angle is not set sufficiently, so after the Fourier transform, the two +1 order spectrums of the object are very close to each other. We used the same filter window to extract the object spectrum, and both the two +1 order spectrums are selected. We found this "mistake" untill the computer run the autofocusing criterion onto the reconstruction process. One of the spectrums became a very serious stripe interference, however, our criterion still achieved the correct result. Unfortunately, the other criteria failed seriously in the reconstruction process. The results are shown as follows:
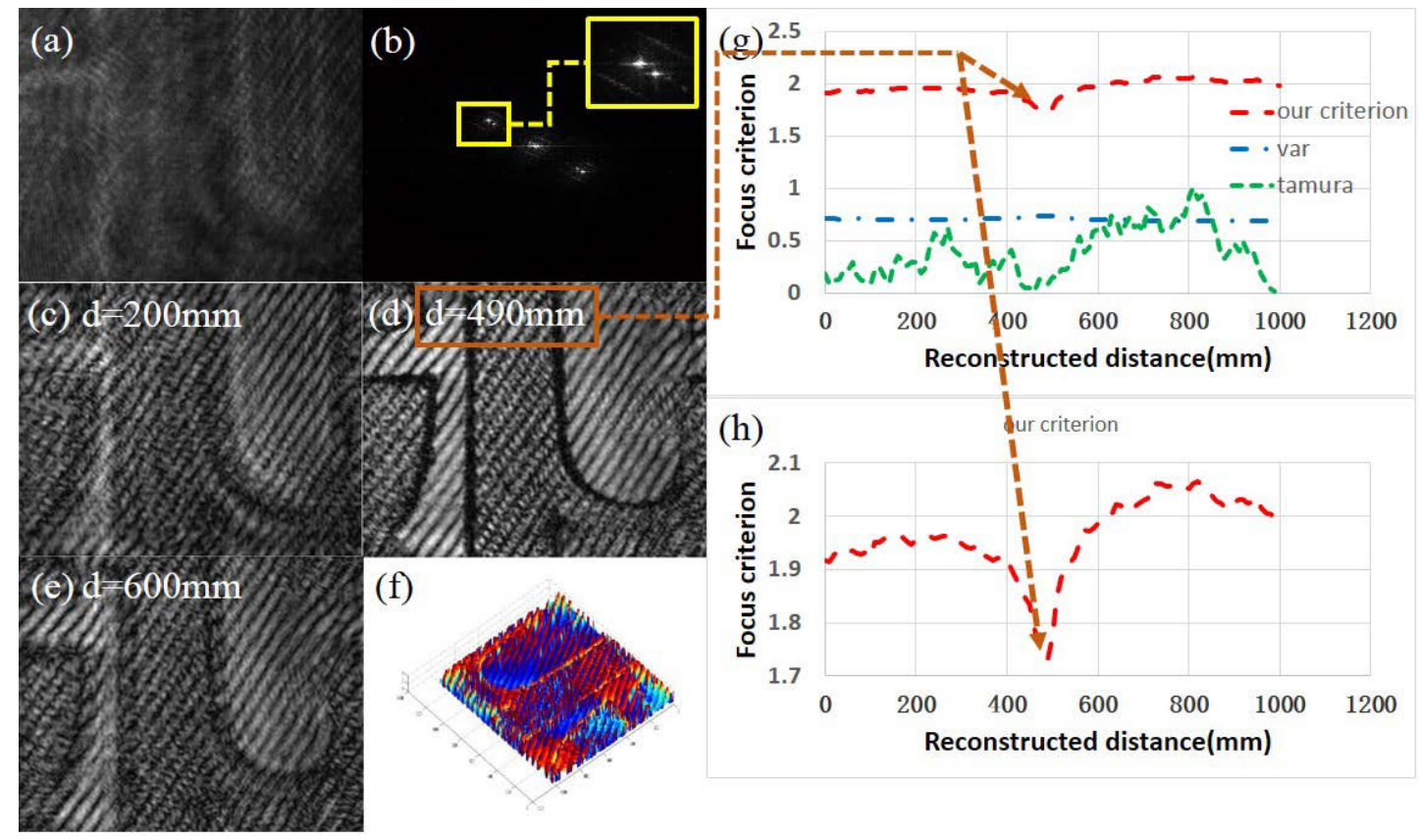

Fig.6 comparison between our criterion and the proposed methods using strong interferenced low quaility hologram. (a)original hologram; (b)Fourier transform of the original hologram; (c),(d),(e) reconstruction processes under different distances; (f)Pseudo-three-dimension plot of the phase of the reconstruction image of (d); (g)three autofocusing criterion curves with different methods; (h)curve detail of our criterion

Fig.6 demonstrates the strong adaptability of our criterion. When the hologram contains a high level of interference, the "tamura coefficient" and "VAR index" both failed. Though the "tamura coefficient" can come up with a peak in fig.5(g), it becomes totally chaotic in fig.6(g). The hologram with a destructive interference ruins all the autofocusing criteria based on mean value calculations. But with our method, the high frequency component of the stripes shown in fig.6(c),(d),(e) is considered 
just as slight interference. Though the high frequency component of the stripes will be focused before, along, or after the object's reconstruction process, its energy still cannot be compared to the high frequency energy of the object. So according to fig.6(g), the criterion curve of our method barely changes compared to that shown in fig.5(g).

Next, we will give demonstration of the speed increase of our method. The laptop we used has a i5-6300U CPU with the main frequency of $2.50 \mathrm{GHz}$ and $8 \mathrm{G}$ ram. We conduct all three methods mentioned in this paper to determine their calculation time with the different qualities of holograms. Table 2 shows the results.

Table 2 the speed of determining the autofocusing time(ms)

\begin{tabular}{|l|l|l|l|}
\hline & Fig.4 & Fig.5 & Fig.6 \\
\hline VAR & 0.3413 & 1.7744 & -- \\
\hline Tamura & 0.3677 & 2.1653 & -- \\
\hline Our method & 0.2195 & 0.6285 & 0.9372 \\
\hline
\end{tabular}

It can be seen from table 2 that, our method can greatly increase the speed of autofocusing. When dealing with the good quality holograms, the speed of these three method are almost the same. However, when dealing with the bad quality of holograms, the advantage of our method appears clearly. When dealing with fig.6, both VAR and Tamura method are failed to reach the conclusion while our method still works effectively.

\section{Conclusions}

We present an autofocusing method along with its criterion in this paper. Our aim is to make the autofocusing process of infrared digital holography more adaptable, stable, and controllable. The method proposed in this paper minimizes the mean value drifting problem that exists in previously proposed methods, and it uses only the high frequency energy of the original hologram to calculate the criterion. This method works as well as other published methods with high quality holograms, and much better and more effective with low quality holograms. Even when the original hologram is degraded by destructive interference, our method can still perform well. 
We are sure that our research will help in developing autofocusing technology of digital holography, and brings this technology out of the lab environment into the real world industrialization.

\section{Acknowledgments}

This work was supported in part by the National Science Foundation of China under Grant 61505083 and the Scientific Research Foundation of Nanjing University of Posts and Telecommunications (NO. NY215043).

\section{References}

1. T. Kim and T. C. Poon, “Autofocusing in optical scanning holography”, Appl. Opt. 48(34), pp.153-159 (2009).

2. Y. Yang, B. S. Kang, and Y. J. Choo, "Focal plane location in digital holography”, Proc. SPIE, 6723, 672365, doi: 10.1117/12.787618 (2007).

3. F. C. Groen, I. T. Young, and G. “A comparison of different focus functions for use in autofocus algorithms”,Lightart, Cytometry 6,81 (1985).

4. Y. Sun, S. Duthaler, and B. J. Nelson, “Autofocusing in computer microscopy: selecting the optimal focus algorithm”, Microsc. Res. Tech.65, 139 (2004).

5. J.-M. Geusebroek, F. Cornelissen, A. W. M. Smeulders, and H. Geerts, "Robust autofocusing in microscopy”, Cytometry 39, 1 (2000).

6. M. T. Özgen and T. E. Tuncer, "Object reconstruction from in-line Fresnel holograms without explicit depth focusing”, Opt. Eng. 43(6), pp.1300-1310 (2004).

7. S. Lee, J. Y. Lee, W. Yang, and D. Y. Kim, “Autofocusing and edge detection schemes in cell volume measurements with quantitative phase microscopy”, Opt. Express 17(8),pp.6476-6486 (2009).

8. L. Yu and L. Cai, "Iterative algorithm with a constraint condition for numerical reconstruction of a three-dimensional object from its hologram”,J. Opt. Soc. Am. A 18(5), pp.1033-1045 (2001).

9. F. Dubois, C. Schockaert, N. Callens, and C. Yourassowsky,” Focus plane detection criteria in digital holography microscopy by amplitude analysis”, Opt. Express 14(13), pp.5895-5908 (2006). 
10. M. Antkowiak, N. Callens, C. Yourassowsky, and F. Dubois,” Extended focused imaging of a microparticle field with digital holographic microscopy”, Opt. Lett. 33(14), pp.1626-1628 (2008).

11. L. Ma, H. Wang, Y. Li, and H. Jin, "Numerical reconstruction of digital holograms for three-dimensional shape measurement”J. Opt. A: Pure Appl. Opt. 6(4),396 (2004).

12. M. Tachiki, M. Itoh, and T. Yatagai, "Simultaneous depth determination of multiple objects by focus analysis in digital holography”, Appl. Opt. 47(19), pp.D144-D153 (2008).

13. W. Li, N. C. Loomis, Q. Hu, and C. S. Davis, "Focus detection from digital in-line holograms based on spectral $l_{1}$ norms” J. Opt. Soc. Am. A 24(10), pp.3054-3062 (2007).

14. P. Memmolo, C. Distante, M. Paturzo, A. Finizio, P. Ferraro, and B. Javidi, "Automatic focusing in digital holography and its application to stretched holograms”, Opt. Lett. 36(10), pp.1945-1947(2011).

15. P Memmolo, M Paturzo, B Javidi, PA Netti, P Ferraro,” Refocusing criterion via sparsity measurements in digital holography”, Opt. Lett, 39(16),pp.4719-4722(2014).

16. Hazar A. Ilhan, MertDogar, Meric- Ozcan, "Fast autofocusing in digital holography using scaled hologram”, Opt. Comm. 287, pp.81-84(2013).

17. Peng Gao, Baoli Yao,* Junwei Min, Rongli Guo, Baiheng Ma, Juanjuan Zheng,Ming Lei, Shaohui Yan, Dan Dan, and Tong Ye, “Autofocusing of digital holographic microscopy based on off-axis illuminations”, Opt. Lett. 37(17), pp.3630-3632(2012). 